\title{
Real Time Data Acquisition and Network Topology Research of Wireless Sensor Based on RFID
}

\author{
https://doi.org/10.3991/ijoe.v14i05.8600 \\ Jing Chen \\ Inner Mongolia University of Science and Technology, Baotou, China \\ jingcheninner@163.com
}

\begin{abstract}
In order to improve the warehouse cargo flow and detect the temperature and humidity of storage, the characteristics of the topology of various wireless sensor networks were compared. The tree topology was used. The routing algorithms and routing methods were summarized. A brief description of the networking process was given. Then, the goods data of wireless sensor network and the data collection process of storage environment were designed. The various nodes and the workflow of the RFID system were described. Finally, the warehousing goods data and environmental data were collected and tested. The results showed that the system accurately identified the pallet tag and the cargo tag. At the same time, the storage temperature and the humidity data were monitored in real time. Therefore, the system meets the company's functional requirements for information collection and environmental monitoring of warehousing goods. The effect is good.
\end{abstract}

Keywords-RFID; wireless sensor; real-time; data acquisition; network topology

\section{Introduction}

Because of its non-contact, fast recognition speed, strong anti-interference ability, long identification distance and high recognition accuracy, RFID technology has been applied in the field of traffic management, automatic charging, logistics and anticounterfeiting. The RFID technology is applied to storage management, and the warehousing goods information is collected and processed in real time. Combined with the wireless sensor network, the storage environment information is monitored. Automated and intelligent warehouse management is realized. While enhancing the efficiency of enterprises, it also meets the development needs of modern warehouse management.

In order to meet the needs of the development of modern logistics, warehousing must adopt intelligent, automated and networked management. An advanced and efficient warehouse management system has an important role in the logistics industry. Based on RFID technology, and combined with wireless sensor network (WSN) technology and database technology, an accurate and efficient warehouse management platform is designed. The collection, transmission, storage and management of 
warehousing materials and storage environment data are integrated. The automation of low-cost warehousing information is implemented.

Using the reader and the electronic label, the RFID system is built to collect the storage material data. The dynamic change of materials in the storage process is controlled efficiently, accurately and safely. The WSN system is constructed by using sensor nodes, to realize the collection of temperature and humidity data of the warehouse. The RFID system and the WSN system are integrated to achieve the real time and effective management of the warehouse goods and warehouse environment. The composition of each part of the system is realized according to the actual demand of storage management. The fixed RFID reader is installed in the forklift and the warehouse exit. The RFID tag is installed on the tray and storage materials. Then, according to the corresponding software, the management of information, such as the quantity of materials and the state of warehousing, is completed.

\section{State of the art}

The developed countries such as the United States, Germany, Japan and other developed countries have begun to pay attention to the development of logistics and storage at the beginning of the last century. Bou-El-Harmel, A. [1] studied the quasi isotropic radiation pattern optimization of the 3D stereoscopic antenna UHF RFID, the wireless sensor network and the application of signal to noise ratio. Enriquez, G. [2] applied wireless sensor networks to the perception of navigation systems. Mohammed, M. [3] used RFID and wireless sensor networks to design an intelligent University. In 1950s, the United States set up special agencies to conduct systematic research on logistics management, and launched WMS (Warehouse Management System) in 1974. It further promots the rapid development of logistics warehousing management. In the next few decades, more and more computer technology and simulation technology are applied to logistics and storage management. Djenouri, D. [4] showed the dramatic impact of mobile metallic objects over the mote on wireless communication channels, which is a typical situation that takes place when detecting a vehicle that parks over the sensor mote. The focus of development has been oriented to the professionalization and standardization of management. The scope of the research is further extended to the whole process from product procurement to sales. Lansey, K E. [5] discussed the evolution towards the first integrated radio-frequency identification (RFID)-enabled wireless sensor network infrastructure using ultra-high frequency/radio frequency sensor nodes and inkjet-printed electronics technologies on flexible and paper substrates for the first time ever.

In developed countries, many technologies are used in warehouse management, including bar code, GPS and RFID. Different technologies have different features, which are suitable for the needs of different warehouse management systems. Among them, RFID technology has been widely studied and used because of its large capacity, strong anti-interference ability, moderate cost and uneasy damage. Palinkas, L A. [6] pointed out that environmentally friendly conformal materials should be used in order to minimize impact on environment as a result of used chemicals or materials in 
the fabrication process as well as RFID disposal. The United States Wal-Mart Store Inc began to use RFID electronic labels in warehousing goods management since 2003. Subsequently, FedEx, UPS and other companies began to introduce RFID technology into its logistics management. In recent years, with the rapid development of Internet of things technology, modern warehouse management system has been widely used in developed countries. The storage management technology is quite mature, and the equipment is more advanced. Quwaider, M. [7] thought that the cost needs to be extremely low, especially for the RFID tagS' mass production, as well as for the existing ID infrastructure modification.

After the use of the RFID system in the United States, the cost of stolen goods was significantly reduced by 2 billion dollars a year, and the competitiveness was rapidly increasing. Metro, Germany, has provided the leading RFID testing device in the industry since 2004, which has promoted the wide application of RFID in the German retail industry. The BestBuy company in the United States began to equip RFID electronic labels with all commodities since 2006. The supply chain efficiency in 5 years has been significantly improved. The results of Malathi, L. [8] studies reveal that there are several advantages of the RFID technology. The no contact and non-line ofsight nature of this technology are significant advantages common among all types of RFID systems. Dong, M. [9] pointed out that all RF tags can be read despite extreme environmental factors, such as snow, fog, ice, paint, and other visually and environmentally challenging conditions. They can also work at remarkable speeds. Velmani, R. [10] proposed an efficient cluster tree-based data acquisition scheme for large mobile wireless sensor networks. Currow, D C. [11] pointed out that under different routing modes, the routing algorithms adopted are also different. In some cases, tags can be read in less than 100 milliseconds. The other advantages are their promising transmission range and cost-effectiveness.

To sum up, the traditional methods cannot improve the warehouse cargo flow and detect the temperature and humidity of storage. To solve this problem, the goods data of wireless sensor network and the data collection process of storage environment were designed. The various nodes and the workflow of the RFID system were described. The warehousing goods data and environmental data were collected and tested. The designed system accurately identified the pallet tag and the cargo tag. At the same time, the storage temperature and the humidity data were monitored in real time. Therefore, the system meets the company's functional requirements for information collection and environmental monitoring of warehousing goods.

\section{$3 \quad$ Methodology}

The storage temperature and humidity environment information detection function need to set up each wireless sensor node into a certain network structure. The network topology, protocol standard and routing interact with each other, which directly affects network startup, allocation mechanism and routing. It plays a vital role. The Zig Bee/IEEE802.15.4 protocol is used to define the physical layer and the MAC layer specification. The physical layer is responsible for channel energy detection, selection 
of channel frequency, transmission and reception of data, activation and deactivation of wireless transceivers. The MAC layer is mainly responsible for providing an interface to the physical channel of high level access.

\subsection{Analysis of ZigBee network topology}

In ZigBee network topology, the role of the coordinator is to form a network when the node is added, and the related operation is carried out after the network is started. The role of routers is to manage data transmission among nodes, as well as to expand the network. Dong, M. [9] pointed out that terminal nodes generally do not have communication functions, and can only join the network. In order to improve the efficiency and accuracy of information transmission in network and prolong the normal operation life of network while reducing network load, the problem of network topology must be well solved.

ZigBee mainly defines the network, security and application framework. Generally, the network layer supports three topologies of star, mesh and cluster tree. Velmani, R. [10] studied the composition and function of different topological structures. Among them, the structure of the star network is the simplex. It saves energy. The service life of the network is long. However, the communication between the nodes must be implemented by the coordinator. The performance of the network is greatly restricted. Mesh networks can be selected flexibly in multiple information transmission paths according to actual needs. Each routing node can communicate with each other directly, and the reliability is high. Clustered tree networks combine star and mesh. It saves energy and has high reliability. The network is also broader.

Topology has a crucial impact on the coverage of the entire ZigBee network, the ability to collect and transmit data, the working energy of the nodes and the working life of the network. Based on the analysis of the characteristics of three topological structures, and according to the design requirements of this system, the ZigBee network is constructed by selecting cluster tree topology. The network topology is shown in Figure 1.

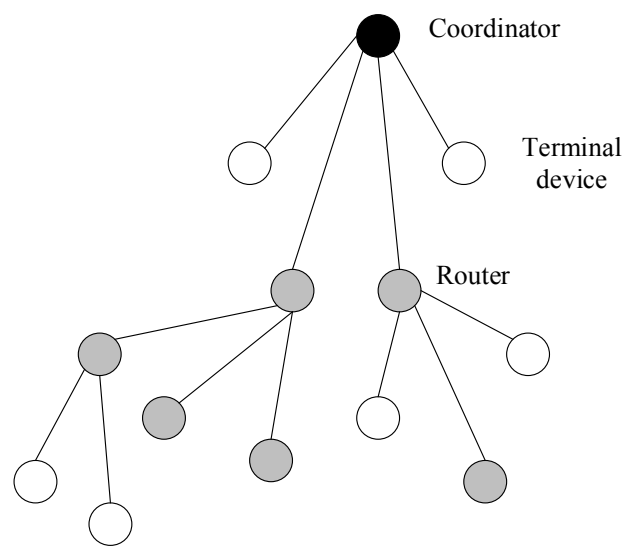

Fig. 1. Tree topology diagram 


\subsection{Implementation of ZigBee network routing}

The routing process is as follows: first, the device sends out the routing request frame and starts the routing discovery. The receiving device then replies to the reply frame after accepting the command. Finally, the optimal route is added to the routing table of each device in the path by comparing the available paths.

The setting of the route usually includes three modes: disallowed route, enabled route and forced route. Among them, in the banned routing mode, if the network router is found, the packet will point to the router; otherwise, it will continue to advance along the tree. In routing mode, the packet also selects the route to communicate when the network router is found. Once the network router is not determined, the router will start the routing discovery process and transmit the packet according to the calculated route after the discovery is completed. If the router does not have routing discovery ability, the packet continues to move along the tree. In the mandatory routing mode, if the router has the capability of routing discovery, the routing discovery process will be forced to start. After the discovery is completed, the data packet is transmitted according to the calculated route. If the router has no routing discovery capability, the packet advances along the tree.

The data frame received through the network layer can determine its source layer and carry out the corresponding routing. The broadcast frames of the MAC sublayer can be forwarded directly and processed in the application layer. The data frames on the MAC sublayer can be determined by comparing the logical address and the destination address. When the address is the same, it does not need to be forwarded to the upper processing. If the address is different, it continues to forward.

The commonly used routing algorithms in ZigBee network include AODV, ADOVjr and Cluster-Tree. In the ZigBee network of cluster tree topology, the routing algorithm combined with tree cluster algorithm and AODVjr algorithm is usually used. In the forbidden routing mode, tree clustering algorithm can only be used to transmit messages along tree topology. The algorithm is combined with tree cluster algorithm and AODVjr algorithm in energy routing mode, and the routing algorithm is selected according to the actual situation. The AODVjr algorithm needs to be used in the mandatory routing mode. If the device has the ability to find the path, it does not need to consider whether the transmission path exists. The path lookup process is directly forced to start. Packets are transmitted along the calculated path when the lookup is completed.

$\mathrm{C}_{\mathrm{m}}$ is the maximum number of sub nodes. $\mathrm{R}_{\mathrm{m}}$ is the largest number of routing nodes in a child node. $L_{m}$ is the maximum network depth. The address space $C_{\text {skip }}(d)$ that the routing node with network depth $\mathrm{d}$ can allocate satisfies the formula (1):

$$
C_{\text {skip }}(\mathrm{d})=\left\{\begin{array}{l}
1+C_{m} \times\left(\mathrm{L}_{\mathrm{m}}-\mathrm{d}-1\right), \mathrm{R}_{\mathrm{m}}=1 \\
\frac{1+C_{m}-R_{m}-C_{m} \times R_{m}{ }^{\left(\mathrm{L}_{\mathrm{m}}-\mathrm{d}-1\right)}}{1-R_{m}}, \text { otherwise }
\end{array}\right.
$$

The address $A_{k}$ assigned to the kth child router node satisfies the formula (2): 


$$
A_{k}=A_{\text {father }}+C_{\text {skip }}(\mathrm{d}) \times(\mathrm{k}-1)+1
$$

The address $A_{n}$ assigned to the nth terminal node satisfies formula (3):

$$
A_{n}=A_{\text {father }}+C_{\text {skip }}(\mathrm{d}) \times R_{m}+n
$$

In the formula, $\mathrm{A}_{\text {father }}$ represents the address of the father node that assigned the network address.

\subsection{Node networking process}

The safety management of storage environment is a very important link in the logistics and warehousing industry. Especially for some storehouses, such as inflammable and explosive materials, fuel powder, gasoline or humidity sensitive, moldy goods, the demand for storage environment safety is more severe. WSN technology has the advantages of convenient networking, no wiring, simple debugging, large monitoring area and extensible. It is very necessary to use it in storage management, to monitor and control the storage environment in real time and effectively. To a large extent, it can prevent accidents from occurring, guarantee the safety of warehouse management, and also meet the needs of modernization, automation and intellectualization of warehousing management. The main business of a logistics company in Fujian Shishi is to store nonflammable and explosive goods. Therefore, it is necessary to realize the monitoring of the storage temperature and humidity environment data in the design. There are a large number of different types of sensor nodes in the WSN monitoring system used in this study. The network node is used to collect the original data. The coordinator node is used to build and start the network and to store and analyze the data. The management node is used to collect, store, process the real time data and check the historical data. Routing nodes are used for processing and sending data.

The networking process is shown in Figure 2. Each node can automatically complete the setting according to the relevant requirements during the networking process.

First, nodes are initialized, including the establishment and initialization of coordinator, initialization of protocol stack for physical layer and MAC layer, and the setting of network address and identifier. Next, through the scan, the appropriate channel is found and the network is started. Finally, the nodes are added to the network and assigned to them to perform the required tasks. 


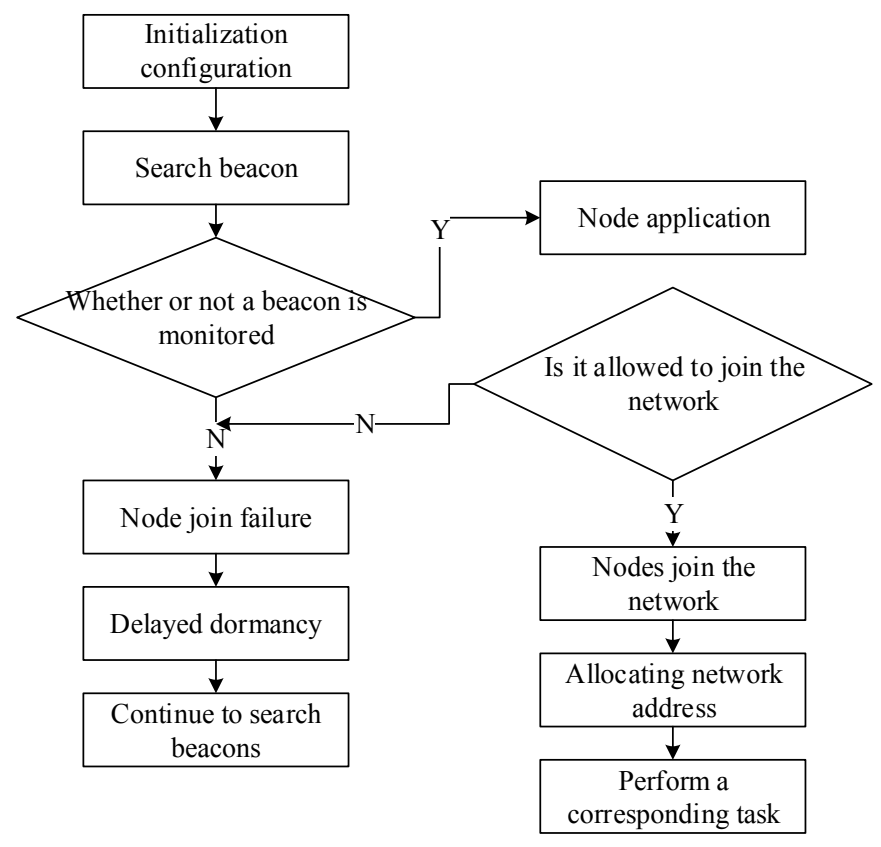

Fig. 2. Node network flow chart

\section{Design of data acquisition module}

\subsection{Data collection process of goods}

Inventory is an important part of storage management. In the past, the accuracy of the inventory was not guaranteed, and it was a waste of time. The efficiency is low. In this design, inventory personnel use a mobile reader to scan the goods and store the relevant information. Then, the scanned data is compared with the storage information stored in the database. Finally, the results are summarized through the report to ensure the accurate management of the warehousing materials.

The customer order is the beginning of the whole warehouse management process, and the subsequent management process should be based on this. The processing flow is shown in Figure 3.

After the order is recorded, it is marked as state 1 . Then, the order is audited. If unqualified, it is marked as state 0 and returned to the customer. If qualified, the specific operation is carried out according to the change state of the number and type. If no change is needed, the delivery plan is made directly; otherwise, the type and quantity of the order should be changed. It is marked as state 2 . After that, the delivery plan is set up. Finally, operation is carried out according to whether the shipment plan changes. If there is no change, it is marked as status 3 and waiting for shipment. If there is any change, then the quantity, type and date of shipment need to be changed before waiting for shipment. 


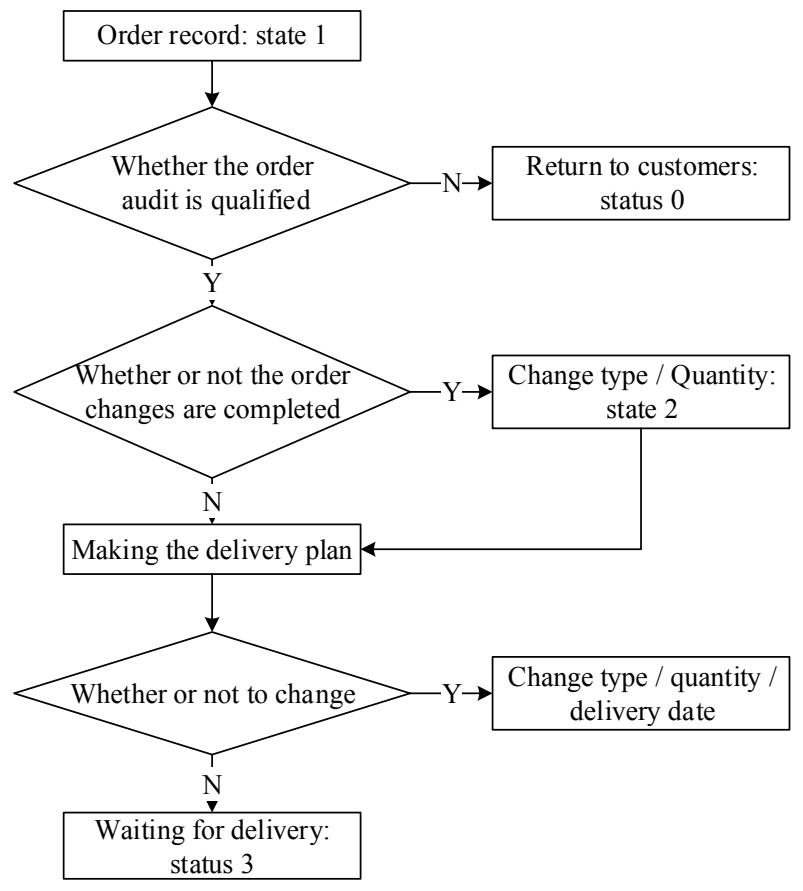

Fig. 3. Order processing flow

The management of outgoing storage is of great significance in the whole warehouse management process. When the goods enter the warehouse, the system will automatically generate a single storage and distribution of goods, determine the cargo storage area, and guide the forklift to finish goods shelves. The cargo pallets are placed in the designated position of the system. Under certain conditions, when the goods cannot be stored in the specified location, location adjustment can be temporarily adjusted. The reader will send the information of the goods to the warehouse management database to update, to ensure the accuracy of inventory information. When warehousing goods are transferred out, according to the order number, the operator can inquire the location of the goods and produce the warehouse receipt. Then the goods were put down. In addition, the reading and writing device on the forklift can also be used to read the information of the goods out of the warehouse. Then through the reader and writer scanning in the warehouse export, the information of the goods out of the warehouse is transmitted to the warehouse management database.

This design uses the RFID system to collect the storage information of the goods in real time. In the management database, the operation of the addition and deletion of the goods is carried out according to the condition of the delivery of the goods. This ensures the real-time and consistency of the information. The generated reports can be used for subsequent queries, calls, and other operations. The basic information of the goods is checked at the beginning of the storage, and then written to the RFID tag installed on the pallet. The RFID reader installed on the warehouse entry will scan the 
checked goods labels and check the information. If a mistake is found, the cargo information is reentered. If the check is not correct, the goods information will be added to the management system database in real time. When the goods are out of the warehouse, the RFID reader and write device of the warehouse gate is also used to scan and read the label of the goods. If a mistake is found, the warehouse manager will be required to deal with it. If it is confirmed, the goods can be deleted directly and the electronic label can be removed.

\subsection{Design of data collection process for storage environment}

The wireless network is constructed by routing nodes, coordinating nodes, terminal nodes and so on. Then, combined with PC, the environment monitoring system is formed. By arranging the WSN nodes in the warehouse, the temperature and humidity data of the storage environment are monitored and collected in real time and transmitted to the information management system by wireless mode. At the same time, it can also control the working state of the network flexibly, so as to achieve the required control requirements. The system can display the collected data by means of numbers and graphics, which is convenient for managers to record, analyze and process effectively. Once the temperature and humidity data monitored by the system are higher than the safety threshold, it will remind the warehouse personnel by the alarm sound. Meanwhile, the red alarm and flashing pictures will appear at the abnormal nodes in the system monitoring interface, which provides solid guarantee for the safety management of storage goods. In order to ensure the working life and reliability of nodes, the system will monitor every node's voltage in real time, and automatically assign tasks of each node according to the detected data, so that the node's working state can be automatically switched between startup and sleep. In addition, in order to improve the collaboration performance and data detection accuracy of all sensors in the network, redundant information processing and complementary processing methods are used to get complete and accurate information.

Based on the ZigBee protocol stack, the software process and program of the JN5139 node module are designed in combination with the Code:: Blocks development tool. The main program flow of the terminal device node is shown in Figure 4.

First, the hardware and software parts are initialized after the node is started. Next, the available network is added to query and process related events. If there is no event that needs to be processed, the node enters a dormant state until it is interrupted before returning to the state of normal work. 


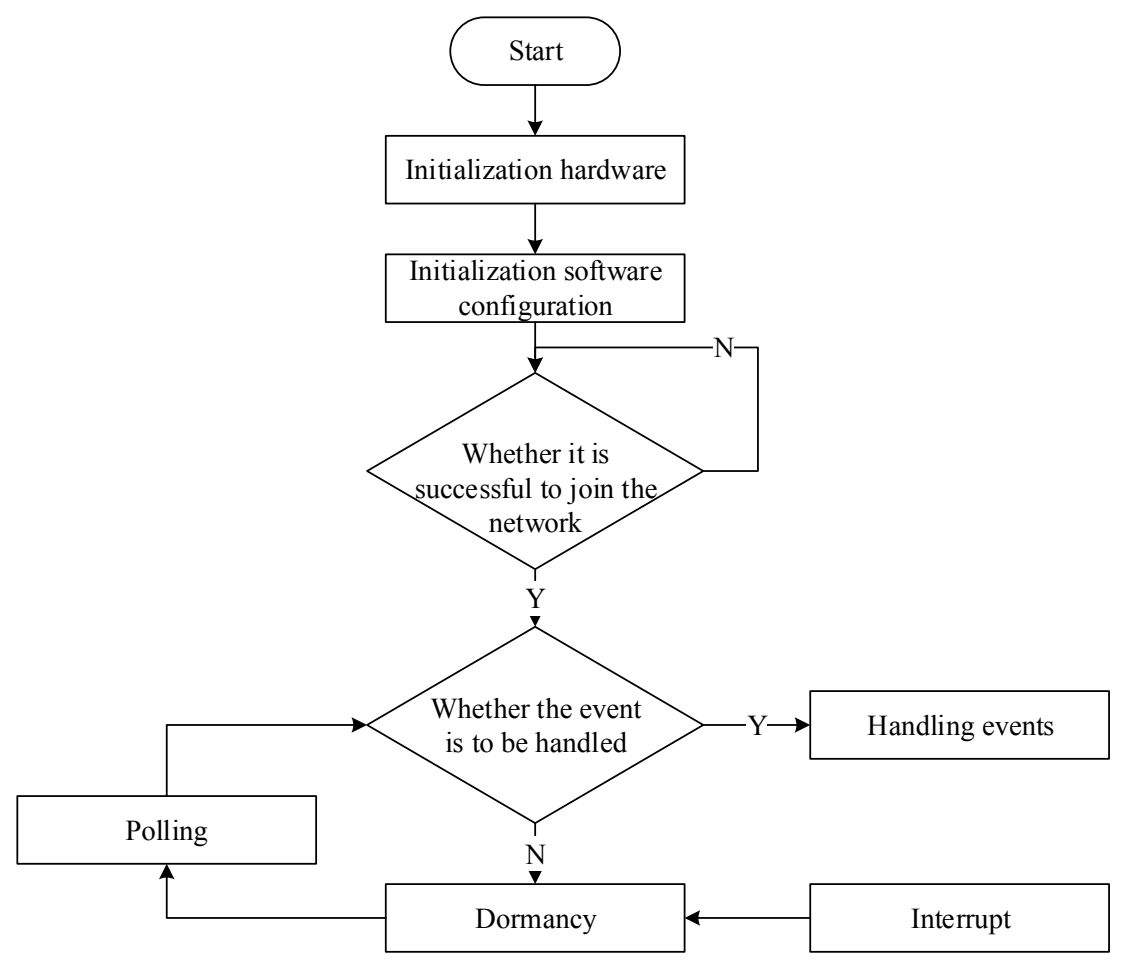

Fig. 4. Terminal device node main program flow

\subsection{Software design of data transmission module}

The coordinator first initializes the hardware and software to prepare the network. Then, by sending a data frame, the channel is scanned in sequence. When the data frame response is obtained, the channel is determined to be empty, or the next channel will continue to be scanned. After the channel is confirmed, the coordinator can listen to it through the network address. If the node of the non-conforming device is applied to join, it will assign a specific network address to it. In the network, the coordinator node is used to aggregate and transmit all the detected data to the information monitoring system. For some node requests that cannot be processed in time, the coordinator will give up. After the node sends a request, if the coordinator's response cannot be obtained, the request will be sent back to the response position. Thus, the coordinator could not get dormant. It is always under working conditions.

The routing nodes in the WSN network can take advantage of their own sensor devices to collect data near the node. According to the actual needs, the collected data are processed by fusion. The function of transmitting data between nodes is achieved by forwarding packets. 


\section{$5 \quad$ Result analysis and discussion}

\subsection{Data acquisition and test results and analysis of warehousing goods}

The test process is as follows. First, the RFID device is used to build a test system. Then, according to the company's actual situation with the trailer storage warehouse process, and considering the position of the label, robustness and recognition distance, environmental factors and so on, the pallet label on the trailer and the cargo label were conducted several rounds of reading test. Take the tray label test results as an example: 15 nonmetal labels are attached to the cargoes loaded on the trailer tray. At the gate of the warehouse, 13 of the tags can be read effectively. After changing the warehouse's temperature and humidity environment data, it is tested again according to the above methods. The tags that can be effectively read are still the 13 ones before, but the number of tags being read successfully is less than before. The test results show that the location of the label has a great influence on the data collected by the RFID system. The temperature and humidity environment factors also affect the reading data. After comparing the results of multiple rounds of tests, it can be concluded that the most effective way to paste the electronic labels on the bottom of the tray is not only to ensure the reliability of data reading, but also to identify easily in software.

\subsection{Data acquisition and test results and analysis of storage environment}

The testing process is carried out in the laboratory. The specific process is as follows. ISnamp-J visual monitoring software and Code:: Blocks program download software is installed on the PC machine, as a test host computer. Then, using the same method, cargo label is tested. The results are basically the same. The location of the label, the temperature and humidity environment and other factors have some influence on the data acquisition system. This system can accurately identify and read on pallet label and cargo label written, which meets the functional requirements of the warehousing goods information collection. 5 nodes are placed in a certain area of the laboratory. Next, the node power is turned on. The iSnamp-J software can be used to observe the state of the nodes, the monitoring data of ambient temperature and humidity, and the working voltage of the nodes, as shown in Figure 5.

From the experimental data, it is found that the temperature values of each node are relatively close, and the humidity is slightly different. In order to further verify the monitoring performance, the area of node No. 2 is heated by the dryer in the experiment. At this time, the test data of the node changes obviously, the temperature value rises to $30^{\circ} \mathrm{C}$. The humidity value also changes. The test results show that the system can achieve real-time monitoring and storage of temperature and humidity data. The effect is good.

The working voltage of each node can be examined by using the temperature curve of the nodes measured in a certain period of time, as shown in Figure 6. It can be seen from curve that the temperature change of node 5 always stays in a certain area. Only 
because of the need of transmission and processing information between nodes, there is slight fluctuation along with the change of node voltage.

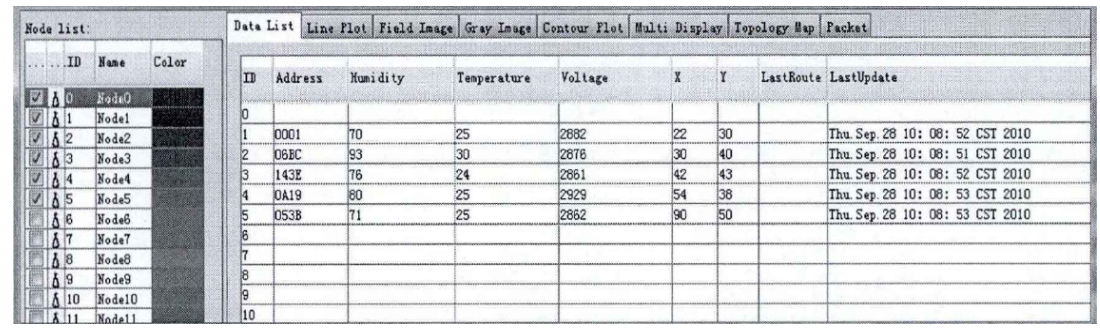

Fig. 5. Warehouse environment data acquisition test chart

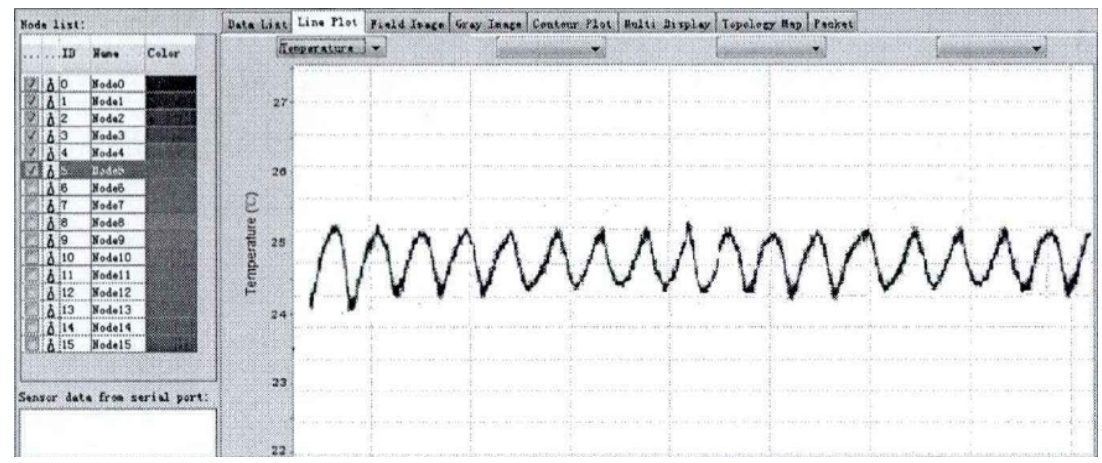

Fig. 6. Temperature collection curve of WSN node

\section{Conclusions}

Based on RFID technology, and combined with wireless sensor network technology, a storage management system is designed. Specifically, the main contents and contributions are as follows:

1. The characteristics of three topological structures, such as star, mesh and tufted tree, are analyzed. The tree topology is used in the system. The routing algorithms and routing methods are summarized. A brief description of the networking process is given.

2. The collection process of the goods data and environmental data is designed. The software design of the router and coordinator in the data transmission module is carried out.

3. The RFID system and the WSN system are tested, and the test results are analyzed. The results show that the automatic monitoring and management of warehousing goods and storage environment is realized in this study. The system has low cost, low power consumption, high efficiency, simple operation and strong expansibility. It meets the needs of the company's business development. 


\section{$7 \quad$ References}

[1] Bou-El-Harmel, A. Optimization of a 3D UHF cubic antenna with quasi-isotropic radiation pattern for RFID, WSN and RSN applications. Wseas Transactions on Communications, 2015, vol. 14, pp. 365-373.

[2] Enriquez, G. Contextual Awareness in a WSN/RFID Fusion Navigation System. Isrn Otolaryngology, 2014, pp. 1-10. https://doi.org/10.1155/2014/198569

[3] Mohammed, M. Designing smart university using RFID and WSN. International Journal of Computer Applications, 2015, vol. 112(15), pp. 34-39.

[4] Djenouri, D. Networked Wireless Sensors, Active RFID, and Handheld Devices for Modern Car Park Management: WSN, RFID, and Mob Devs for Car Park Management. International Journal of Handheld Computing Research, 2015, vol. 6(3), pp. 33-45. https://doi.org/10.4018/IJHCR.2015070103

[5] Lansey, K E. Discussion of "Calibration Assessment and Data Collection for Water Distribution Networks". Journal of Hydraulic Engineering, 2015, vol. 127(4), pp. 270-279. https://doi.org/10.1061/(ASCE)0733-9429(2001)127:4(270)

[6] Palinkas, L A. Purposeful Sampling for Qualitative Data Collection and Analysis in Mixed Method Implementation Research. Administration \& Policy in Mental Health, 2015. Vol. 42(5), pp. 533. https://doi.org/10.1007/s10488-013-0528-y

[7] Quwaider, M. Cloudlet-based Efficient Data Collection in Wireless Body Area Networks. Simulation Modelling Practice \& Theory, 2015, vol. 50, pp. 57-71. https://doi.org/10.1016/ j.simpat.2014.06.015

[8] Malathi, L. Energy efficient data collection through hybrid unequal clustering for wireless sensor networks. Computers \& Electrical Engineering, 2015, vol. 48(C), pp. 358-370. https://doi.org/10.1016/j.compeleceng.2015.06.019

[9] Dong, M. RMER: Reliable and Energy-Efficient Data Collection for Large-Scale Wireless Sensor Networks. IEEE Internet of Things Journal, 2016, vol. 3(4), pp. 511-519. https://doi.org/10.1109/JIOT.2016.2517405

[10] Velmani, R. An Efficient Cluster-Tree Based Data Collection Scheme for Large Mobile Wireless Sensor Networks. IEEE Sensors Journal, 2015, vol. 15(4), pp. 2377-2390. https://doi.org/10.1109/JSEN.2014.2377200

[11] Currow, D C. Improving national hospice/palliative care service symptom outcomes systematically through point-of-care data collection, structured feedback and benchmarking. Supportive Care in Cancer, 2015, vol. 23(2), pp. 307-315. https://doi.org/10.1007/s00520$\underline{014-2351-8}$

\section{Author}

Jing Chen is with Inner Mongolia University of Science and Technology, Baotou Teachers' College, Baotou 014030, China (jingcheninner@163.com).

Article submitted 21 March 2018. Final acceptance 04 May 2018. Final version published as submitted by the author. 